\title{
Role of multi-detector computed tomography in the detection and differentiation of adnexal mass lesions
}

\author{
Ezzat Khalda $^{1 *}$, Hafizur Rahman ${ }^{2}$
}

\begin{abstract}
${ }^{1}$ Department of Radio-diagnosis, Sikkim Manipal Institute of Medical Sciences- Sikkim Manipal University, Tadong, Gangtok, Sikkim, India

${ }^{2}$ Department of Obstetrics and Gynecology, Sikkim Manipal Institute of Medical Sciences- Sikkim Manipal University, Tadong, Gangtok, Sikkim, India
\end{abstract}

Received: 21 April 2019

Accepted: 30 May 2019

\section{*Correspondence:}

Dr. Ezzat Khalda,

E-mail: ezzyhafiz@gmail.com

Copyright: ( ) the author(s), publisher and licensee Medip Academy. This is an open-access article distributed under the terms of the Creative Commons Attribution Non-Commercial License, which permits unrestricted non-commercial use, distribution, and reproduction in any medium, provided the original work is properly cited.

\section{ABSTRACT}

Background: The objective of this study was to evaluate the role of multi-detector computed tomography (MDCT) in the detection and differentiation of adnexal masses using post-operative histopathology findings as the gold standard. Methods: One hundred and forty five cases that were referred with a primary diagnosis of adnexal masses on clinical or USG examination were evaluated by MDCT in the Department of Radiodiagnosis from January 2013 to December 2013. One hundred twelve cases subsequently underwent surgical exploration and histopathological examination, which was used as a control for the evaluation of MDCT findings, were included in this study.

Results: Majority (54.5\%) of the patients were in the age group of 31-50 years. MDCT detected adnexal masses as malignant in 56 cases, while in other 56 cases it read adnexal masses as benign. Final histopathology revealed adnexal masses in $57(51 \%)$ cases as malignant while in $55(49 \%)$ cases as benign. There were three cases which on MDCT appeared as malignant were subsequently found to be benign in histopathology. Similarly there were four cases which on MDCT appeared as benign were subsequently proved to malignant in histopathology. The sensitivity, specificity, Positive predictive value and negative predictive value of MDCT for diagnosing a malignant adnexal mass was 93.0\%, 94.5\%, 94.6\% and 92.8\% respectively. MDCT findings more predictive of malignancy were solid or cysticsolid mass, necrosis in a solid lesion, cystic lesion with thick, irregular walls or septa, and/or with papillary projections. The presence of ascites, peritoneal metastases, and lymphadenopathy were also helpful to confirm malignancy.

Conclusions: MDCT is an excellent and accurate non-invasive modality in the detection and characterization of adnexal masses from benign and malignant.

Keywords: Adnexal mass, Benign, Computed tomography, Malignant, Sensitivity, Specificity

\section{INTRODUCTION}

Adnexal masses represent a spectrum of conditions from gynecologic and nongynecologic sources. They may be benign or malignant. Ovarian cancer is the leading cause of death from gynecologic malignancy. It is the fifth leading cause of cancer death in the United States among female population. ${ }^{1,2}$ The risk of ovarian cancer increases steadily with age with the greatest risk occurring after menopause. There is a 1.42 percent lifetime risk of dying from ovarian cancer. ${ }^{2}$

When ovarian cancer does occur, it tends to do so in prepubescent girls and in post-menopausal women. 
Although most masses in prepubescent girls are benign, 5 to 35 percent are malignant. ${ }^{3-7}$ Small (less than $2 \mathrm{~cm}$ ) functional cysts can develop in newborns, but these are related to maternal hormones and usually regress during the first months of life. ${ }^{4,8}$ Ovarian cancer is rare in premenopausal women. Other etiologies, such as functional cysts, leiomyomata, and ectopic pregnancy, are more common and can cause significant morbidity. In pregnant women, the most common cause of an adnexal mass is a corpus luteum cyst. In nonpregnant patients, the most common etiologies are functional cysts and leiomyomata. ${ }^{9}$ In postmenopausal women, 30 percent of adnexal masses are malignant. ${ }^{10}$

The goal of evaluation is to differentiate between benign and more serious conditions, such as ovarian cancer. While the diagnosis may be delayed because of unspecified symptoms, appropriate treatment plan will be achievable with deliberate staging of the tumor and will follow by a better outcome. ${ }^{11}$ The presence of an adnexal mass is the leading indications for gynecologic surgery, but the characterization of clinically diagnosed ovarian masses is frequently not possible until surgery and histopathologic examination have been performed. In most institutions the type of surgery (laparotomy vs. laparoscopy) depends on the probability of malignancy, which is based mostly on imaging appearance. ${ }^{12,13}$

Putting together with a thorough observation, physical examination and characteristics of the mass will give valuable information about its nature. ${ }^{14,15}$ Afterward, several invasive and non-invasive paraclinical evaluations can provide additional information. ${ }^{16,17}$

Computed tomography of abdomen and pelvic can depict the adnexal masses as well as probable local or regional invasions. Additionally, it can differentiate gastrointestinal tract, urinary tract and reproductive malignancy from each other using contrast materials. Multidetector computed tomography (MDCT) makes multiplanar evaluation of pelvic and abdominal structures available as well as two- or three-dimensional illustrations. ${ }^{18}$ Further given details about the extension of the tumors particularly improves the treatment plan and outcome.

The 16-slice MDCT can provide high quality images of surrounding organs like diaphragm, paracolic gutters and intestine which defines patients who will benefit neoadjuvant chemotherapy before debulking. ${ }^{19}$ Although the diagnostic accuracy of spiral CT and its axial views for nature and extension of the adnexal masses are reported, local data regarding mass evaluation by MDCT are scarce. ${ }^{20}$ For better evaluation of adnexal masses to differentiate between benign and more serious conditions, such as ovarian cancer this study was undertaken in the Department of Radio-diagnosis, Patna Medical College Hospital, Patna, with the objectives to evaluate the role of multi-detector computed tomography (MDCT) imaging in characterizing adnexal masses and to co-relate the diagnostic value of MDCT with post-operative histopathology findings in the detection and differentiation of adnexal masses.

\section{METHODS}

This was a prospective hospital based analytic-descriptive study conducted during the period from 1st January 2013 to 31st December 2013 in the department of Radiodiagnosis, Patna Medical College Hospital, Patna, India. All patients with clinically or ultrasonographically detected adnexal masses who were referred from Obstetrics and Gynaecology department for MDCT evaluation and subsequently underwent surgical exploration and histopathological examination were included for this study. One hundred and forty five cases that were referred with a primary diagnosis of adnexal masses on clinical or USG examination were evaluated by MDCT in the Department of Radio diagnosis. Excluded from the study were patient with adnexal mass who did not undergo surgical exploration and histopathological evaluation, adnexal masses $<6 \mathrm{~cm}$ with clear cyst in reproductive age women, non gynaecological origin of adnexal masses, Poly Cystic Ovarian Syndrome ectopic pregnancy and critically ill patients. Institutional ethical committee approval was obtained. A written informed consent was obtained from all the participants.

\section{MDCT protocol}

At first patients' brief history and physical examination findings any were noted down from patients' case sheet before MDCT imaging and recorded on a predesigned and pre-tested proforma.

All MDCT were performed on GE Bright Speed 16 Slice CT scan machine in the department of Radiodiagnosis, Patna Medical College Hospital. Image scanning parameters were as follows: rotation time 1 second, table speed $15.4 \mathrm{~mm} /$ rotation, reconstruction interval $0.6 \mathrm{~mm}$ at Kernel H20, $120 \mathrm{kV} / 260 \mathrm{mAs}$, and acquisition time 9s.

MDCT images were obtained from the abdomen and pelvic, covering the area from the diaphragm to the symphysis pubis (craniocaudal). All scans were done with a standard protocol using the triple phase. Precontrast scan of the upper abdomen; arterial phase using the automatic.

\section{Bolus tracking system}

Portal phase yielded with a delay of $60 \mathrm{~s}$ after the arterial one. The contrast medium (iohexol) was administered at a dose of $1.5 \mathrm{~mL}$ per $\mathrm{kg}$, with a variable flow rate of $3-4$ $\mathrm{mL}$ per second through the antecubital vein of the right arm.

To facilitate the differentiation of calcified peritoneal implants from bowel loops, $500 \mathrm{ml}$ of water was 
administrated 30 min prior to the examination. Although it may be difficult to recognize small peritoneal implants and distinguishing them from bowel loops, a careful evaluation of multiplanar reformatted (MPR) images usually enables this differentiation. All patients were fixed during MDCT examination to prevent motion artifacts.

MDCT images were initially interpreted at work station and later on reviewed by an experienced consultant radiologist. MDCT findings used to diagnose malignancy were: diameter greater than $4 \mathrm{~cm}$, presence of masses bilaterally, cystic-solid mass, necrosis in a solid lesion, cystic lesion with thick, irregular walls or septa and/or with papillary projections. Presence of ascites, peritoneal metastases and lymphadenopathy were used to confirm malignancy.

\section{Histopathological co-relation}

An experienced pathologist examined all the resected specimens with no knowledge of the MDCT findings. The histopathological findings were considered as a control for the evaluation of MDCT findings in adnexal mass.

\section{Statistical analysis}

The collected data were thoroughly checked, then entered in an Excel spreadsheet (Microsoft, Redmond, WA, USA) for analysis. The method consisted of transcription, preliminary data inspection, and interpretation. Data were analyzed using GraphPad Instat version 3 (GraphPad Software, La Jolla, CA, USA). Results are presented as number and percentages. The sensitivity, specificity, positive predictive value (PPV) and negative predictive value (NPV) for MDCT were calculated in comparison with histopathological findings.

\section{RESULTS}

One hundred and forty five cases that were referred with a primary diagnosis of adnexal masses on clinical or USG examination were evaluated by multidetector computed tomography (MDCT) of which $112(77.2 \%)$ patients met the inclusion criteria and were considered for final analysis.

Table 1: Age distribution of cases.

\begin{tabular}{|ll|l|}
\hline Age (Years) & Number $(\mathbf{n})$ & Percentage $(\%)$ \\
\hline$<21$ & 8 & 7.1 \\
\hline $21-30$ & 14 & 12.5 \\
\hline $31-40$ & 28 & 25.0 \\
\hline $41-50$ & 33 & 29.5 \\
\hline $51-60$ & 20 & 17.9 \\
\hline$>61$ & 9 & 8.0 \\
\hline Total & $\mathbf{1 1 2}$ & $\mathbf{1 0 0 . 0}$ \\
\hline
\end{tabular}

Among the patients majority $(54.5 \%)$ were in the age group of 31-50 years (Table1). Most common presentation were pain abdomen and/pelvic pain $(76 \%)$ followed by mass abdomen and/distension of abdomen $(59 \%)$. Many patients presented with multiple complaints. Fifteen percent patients' were asymptomatic.

Table 2: Distribution of adnexal masses as benign and malignant as detected by MDCT.

\begin{tabular}{|lll|}
\hline Type & Number (n) & Percentage (\%) \\
\hline Benign & 56 & 50 \\
\hline Malignant & 56 & 50 \\
\hline Total & $\mathbf{1 1 2}$ & $\mathbf{1 0 0 . 0}$ \\
\hline
\end{tabular}

Table 3: MDCT diagnosis of adnexal mass lesions.

\begin{tabular}{|lll|}
\hline Diagnosis & $\begin{array}{l}\text { Number } \\
(\mathrm{n})\end{array}$ & $\begin{array}{l}\text { Percentage } \\
(\%)\end{array}$ \\
\hline Left benign ovarian cyst & 24 & 21.4 \\
\hline $\begin{array}{l}\text { left malignant ovarian } \\
\text { tumour }\end{array}$ & 23 & 20.5 \\
\hline $\begin{array}{l}\text { Right malignant ovarian } \\
\text { tumour }\end{array}$ & 18 & 16.1 \\
\hline Right benign ovarian cyst & 17 & 15.2 \\
\hline $\begin{array}{l}\text { B/L malignant ovarian } \\
\text { tumour }\end{array}$ & 15 & 13.4 \\
\hline Right dermoid cyst & 8 & 7.1 \\
\hline B/L benign ovarian tumour & 4 & 3.6 \\
\hline Left dermoid cyst & 2 & 1.8 \\
\hline $\begin{array}{l}\text { B/L haemorrhagic ovarian } \\
\text { cyst }\end{array}$ & 1 & 0.9 \\
\hline Total & $\mathbf{1 1 2}$ & $\mathbf{1 0 0 . 0}$ \\
\hline
\end{tabular}

Table 4: Distribution of adnexal masses as benign and malignant as diagnosed in histopathology.

\begin{tabular}{|lll|}
\hline Type & Number (n) & Percentage (\%) \\
\hline Benign & 55 & 49.1 \\
\hline Malignant & 57 & 50.9 \\
\hline Total & $\mathbf{1 1 2}$ & $\mathbf{1 0 0 . 0}$ \\
\hline
\end{tabular}

MDCT evaluation revealed adnexal masses were unilateral in maximum $(82 \%)$ cases while in $18 \%$ cases they were bilateral. In most $(51 \%)$ of the cases, adnexal masses were cystic and in $18 \%$ cases adnexal masses were solid, while in $31 \%$ cases both cystic-solid components were observed. Most of the adnexal masses were thin walled $(52 \%)$ having necrosis $(57 \%)$ and absent septation (54\%). Papillary projections in the adnexal masses were observed only in $36 \%$ cases, while in $64 \%$ adnexal masses papillary growths were absent. Thirty four percent patients had ascites. MDCT detected adnexal masses as malignant in 56 cases, while in other 56 cases it read adnexal masses as benign (Table 2). Common MDCT diagnosis of adnexal mass lesion included left benign ovarian cyst $(21.4 \%)$, left malignant ovarian tumour $(20.5 \%)$, right malignant ovarian tumour 
(16.1\%), right benign ovarian cyst $(15.2 \%)$, bilateral malignant ovarian tumour $(13.4 \%)$ and right dermoid cyst (7.1\%) (Table 3).

\section{Table 5: Histopathological diagnosis of adnexal} mass lesions.

\begin{tabular}{|lll|}
\hline Histopathological diagnosis & $\begin{array}{l}\text { Number } \\
(\mathrm{n})\end{array}$ & $\begin{array}{l}\text { Percentage } \\
(\%)\end{array}$ \\
\hline Serous cyst adenocarcinoma & 30 & 26.8 \\
\hline Serous cyst adenoma & 29 & 25.9 \\
\hline Mucinous cyst adenocarcinoma & 17 & 15.2 \\
\hline Mucinous cyst adenoma & 8 & 7.1 \\
\hline Mature cystic teratoma & 10 & 8.9 \\
\hline Malignant dysgerminoma & 5 & 4.5 \\
\hline Paraovarian cyst & 5 & 4.5 \\
\hline Immature teratoma & 4 & 3.6 \\
\hline Endometrioma & 2 & 1.8 \\
\hline $\begin{array}{l}\text { Non-gestational ovarian } \\
\text { Choriocarcinoma }\end{array}$ & 1 & 0.9 \\
\hline Congested serous cyst adenoma & 1 & 0.9 \\
\hline Total & $\mathbf{1 1 2}$ & $\mathbf{1 0 0 . 0}$ \\
\hline
\end{tabular}

Of the 112 cases, final histopathology revealed adnexal masses in $57(51 \%)$ cases as malignant while in $55(49 \%)$ cases as benign (Table 4). The common benign masses included serous cystadenoma (29), mature cystic teratoma (10) and mucinous cyst adenoma (8). The common malignant lesions were serous cyst adenocarcinoma (30), mucinous cyst adenocarcinoma (17) and malignant dysgerminoma (5) (Table 5).

There were three cases which on MDCT appeared as malignant were subsequently found to be benign in histopathology (false positives). One case had solid component with papillary projections and necrosis, while other two had both cystic and solid component with septations and necrosis. All the three cases had ascites as an ancillary finding which lead us to an erroneous diagnosis as malignant.

Similarly there were four cases which on MDCT appeared as benign were subsequently proved to malignant in histopathology (false negatives).

In two cases adnexal masses were thin walled unilocular cystic mass without any evidence of hameorrhage or necrosis were found to be serous cyst adenocarcinoma on histopathology. One well defined adnexal mass with cystic-solid component and fat deposits diagnosed as mature teratoma were found to be immature teratoma in histopathology, while the other cases was found to be a mucinous cystadenocarcinoma on histopathology.

Table 6: Sensitivity, specificity, positive predictive value and negative predictive value of MDCT in the detection and differentiation of adnexal mass lesions.

\begin{tabular}{|llll|}
\hline MIDCT diagnosis & Histopathological diagnosis & Total \\
\hline Malignant & Malignant & 3 & $\mathbf{5 6}$ \\
\hline Benign & 53 & 52 & $\mathbf{5 6}$ \\
\hline Total & 4 & $\mathbf{5 5}$ & $\mathbf{1 1 2}$ \\
\hline & $\mathbf{5 7}$ & $93.0 \%$ & \\
\hline & Sensitivity & $94.5 \%$ & \\
\hline & Specificity & $94.6 \%$ & \\
\hline & Positive predictive value (PPV) & $92.8 \%$ & \\
\hline & Negative predictive value (NPV) & $92.0 \%$ & \\
\hline & False negatives & $7.0 \%$ & \\
\hline & False positive & $5.5 \%$ & \\
\hline
\end{tabular}

The specificity, i.e. ability of MDCT to identify correctly those adnexal masses that are not malignant (i.e. true negatives) was high, $94.5 \%$ in our study. The Sensitivity, i.e. ability of MDCT to identify correctly those adnexal masses which are malignant (i.e. true positives) was $93 \%$ in the present study. Negative Predictive Value, i.e. the probability that a patient with adnexal mass found to be benign on MDCT, in fact will not have malignancy on histopathology was $92.8 \%$ in this study. Positive Predictive Value i.e. the probability that a patient with adnexal mass found to be malignant on MDCT will have, in fact malignancy on histopathology was found to be $94.6 \%$ in our study. False Negatives of MDCT i.e. those cases with adnexal masses which were malignant (on histopathology) but were not detected (i.e. shown as benign) by MDCT was low (7\%) in our study. False Positives i.e. those cases with adnexal masses which were benign (on histopathology) but were shown malignant by MDCT was also low (5.5\%) in this study (Table 6).

\section{DISCUSSION}

An adnexal mass may be found in women of all ages and they are one of the leading causes of death in industrialized countries among female population. Prevalence of ovarian malignancy in prepubertal girls 
varies 5-35\%, while in postmenopausal women approximately $30 \%$ adnexal masses are malignant. . $^{3-7,10}$

Precise characterization of an adnexal lesion is important as it dictates further management; hence, the role of radiology is very important. Until the last decade, exploratory laparotomy was used for the diagnosis and staging of adnexal masses, however, modern imaging techniques have demonstrated similar accuracy in the diagnosing and staging of ovarian carcinoma. Ultrasound is the first line modality to evaluate adnexal pathologies, however, most of the time it is unable to differentiate between benign and malignant lesions and the extent of disease in malignant cases. Sometimes when conventional ultrasound reveals complex morphology then other diagnostic tools can be used. CT is the investigation of choice in planning further management in patients believed to have metastatic disease. Multidetector computed tomography also allows comprehensive evaluation of primary tumour and the site of peritoneal metastasis and lymphadenopathy. On CT scan, masses can be characterized and features pertaining to benignity and malignancy can be observed. ${ }^{21}$

Patients with ovarian cancer present with nonspecific symptoms. The most common symptoms reported by women with ovarian cancer are pelvic or abdominal pain; increased size of the abdomen. ${ }^{1,9}$ In our study $76 \%$ patients presented with pelvic and abdomen pain followed by abdominal distension (59\%). Fifteen percent patients were asymptomatic.

There were three cases which on MDCT appeared as malignant were subsequently found to be benign in histopathology (false positives). Similarly there were four cases which on MDCT appeared as benign were subsequently proved to malignant in histopathology (false negatives).

Several studies have suggested that MDCT can play an important role in characterizing ovarian masses, emphasizing the comparability of CT to other imaging modalities such as magnetic resonance imaging or USG. The sensitivity of morphologic analysis with MDCT in predicting ovarian malignancy has been shown to be 80 $97 \%$, where as its specificity ranged from $85-97 \% .^{22-30}$ The sensitivity and specificity of MDCT for diagnosing a malignant adnexal mass was $93.0 \%$ and $94.5 \%$, respectively in this study compared to gold standard histopathological evaluation of post-operative specimens.

Our results are comparable to previously reported international literature (Table 7). A study conducted by Gatreh-Samani et al, described sensitivity and specificity of MDCT to be $92.8 \%$ and $88.0 \%$ respectively when used for indeterminate masses seen on USG. ${ }^{28}$ Tsili et al, also reported the sensitivity of the16-slice MDCT to be $90 \%$ and accuracy of $89.1 \%$ for detecting malignant tumors in patients with an adnexal mass. ${ }^{23}$ However, higher sensitivity (90.5\%) and accuracy (92.9\%) were reported by the same MDCT imaging method later. ${ }^{25}$

\section{Table 7: Sensitivity, specificity, PPV and NPV of MDCT in differentiation of adnexal masses by different studies.}

\begin{tabular}{|lllll|}
\hline Author & Sensitivity & Specificity & PPV & NPV \\
\hline Byrom et al $^{20}$ & $90 \%$ & $85 \%$ & $85 \%$ & - \\
\hline Tsili et al $^{26}$ & $90.5 \%$ & $93.7 \%$ & - & - \\
\hline $\begin{array}{l}\text { Firoozabadi } \\
\text { et al }\end{array}$ & $79.2 \%$ & $91.6 \%$ & - & - \\
\hline Mubarak et al $^{21}$ & $97.3 \%$ & $91.6 \%$ & $97.3 \%$ & $91.6 \%$ \\
\hline $\begin{array}{l}\text { Gatreh-Samani } \\
\text { al }\end{array}$ & $92.8 \%$ & $88.0 \%$ & $95.5 \%$ & $81.4 \%$ \\
\hline Khattak et al $^{29}$ & $92.0 \%$ & $86.7 \%$ & $94.5 \%$ & $86.7 \%$ \\
\hline Khalda E* $^{*}$ & $93.0 \%$ & $94.5 \%$ & $94.6 \%$ & $92.8 \%$ \\
\hline
\end{tabular}

*Present study

Positive predictive value (PPV) and negative predictive value (NPV) in our study was $94.6 \%$ and $92.8 \%$ respectively. Gatreh-Samani et al, also described $95.5 \%$ PPV and $81.4 \%$ NPV in their study (Table 7). ${ }^{28}$

Our study shows high sensitivity (93\%) and specificity (94\%) of MDCT evaluation of adnexal masses, however, there were three false positive and four false negative results. Lesions characterized as benign have imaging characteristics similar to benign lesions, i.e., smooth walls without thick septations, making evaluation of these tumors difficult. Similarly, regarding false positive results, these lesions have characteristics of malignant lesions, i.e., solid lesions or solid components with necrosis, image appearance of involvement to adjacent organs and the presence of ascites. These features make it difficult to recognize on images, resulting in false positive and negative results. Other possibilities include interpretation error or not using reformatted images properly.

The results of the present study describe the significant agreement between MDCT and histopathology in detection and differentiation of adnexal masses. Also, it has been demonstrated that MDCT have high efficacy and accuracy in defining the nature of a pelvic mass and detecting extension of malignant tumours which could be very useful in planning of treatment.

The sensitivity, specificity, positive and negative predictive value of $\mathrm{CT}$ has some potential limitations which are the topic of today researches to be improved. The sensitivity of CT for detecting peritoneal metastasis is reported about $85-93 \%$, while it decreases to $20-25 \%$ when the metastasis is lesser than $1 \mathrm{~cm}$ in diameter. This may be minimized by thinner slices, loss of artefacts due to partial volume effect and multiplanar reformatting which makes it possible to evaluate bending planes as well. $^{25}$ 
Our study has a few limitations. Besides the small number of samples, only those patients who were referred for MDCT evaluation were included, which introduces bias. No inter-observer agreement for MDCT images evaluation was calculated.

\section{CONCLUSION}

MDCT is an excellent and accurate non-invasive modality in the detection and characterization of adnexal masses from benign and malignant with high sensitivity and specificity. Further research with large number of subject is recommended to remove the potential limitations in detecting smaller peritoneal metastasis and inter-observer agreement evaluation.

\section{ACKNOWLEDGMENTS}

Authors would like to thank all faculties, residents of Department of Radio-diagnosis, $\mathrm{PMCH}$ and all the patients who participated to make this study possible.

Funding: No funding sources

Conflict of interest: None declared

Ethical approval: The study was approved by the Institutional Ethics Committee

\section{REFERENCES}

1. American Cancer Society. Cancer facts and figures 2007. Atlanta, Ga.: American Cancer Society; 2007. Available at: http://www.cancer.org/downloads/ STT/CAFF2007PWSecured.pdf. Accessed on August 3, 2009.

2. Ries LA, Melbert D, Krapcho M, eds. SEER cancer statistics review, 1975-2004. Bethesda, Md.: National Cancer Institute. Available at: http://seer.cancer.gov/csr/1975_2004. Accessed August 3, 2009.

3. Laufer MR, Goldstein DP. Ovarian cysts and neoplasms in infants, children, and adolescents. Up To Date. Available at: http://www.uptodate.com. Accessed August 3, 2009.

4. Schultz KA, Ness KK, Nagarajan R, Steiner ME. Adnexal masses in infancy and childhood. Clin Obstet Gynecol. 2006;49(3):464-79.

5. Cass DL, Hawkins E, Brandt ML. Surgery for ovarian masses in infants, children, and adolescents: 102 consecutive patients treated in a 15 -year period. J Pediatr Surg. 2001;36(5):693-9.

6. Quint EH, Smith YR. Ovarian surgery in premenarchal girls. J Pediatr Adolesc Gynecol. 1999;12(1):27-9.

7. Stepanian M, Cohn DE. Gynecologic malignancies in adolescents. Adolesc Med Clin. 2004;15(3):54968.

8. Sakala EP, Leon ZA, Rouse GA. Management of antenatally diagnosed fetal ovarian cysts. Obstet Gynecol Surv. 1991;46(7):407-14.
9. Stenchever MA. Comprehensive Gynecology. $4^{\text {th }}$ ed. St. Louis, Mo.: Mosby; 2001.

10. Kinkel K, Lu Y, Mehdizade A, Pelte MF, Hricak H. Indeterminate ovarian mass at US: incremental value of second imaging test for characterization metaanalysis and Bayesian analysis. Radiology. 2005;236(1):85-94.

11. Dressman HK, Berchuck A, Chan G, Zhai J, Bild A, Sayer R, et al. An integrated genomic-based approach to individualized treatment of patients with advanced-stage ovarian cancer. J Clin Oncol. 2007;25:517-25.

12. Curtin JP. Management of the adnexal mass. Gynecol Oncol. 1994;55:S42-46.

13. NIH consensus conference. Ovarian cancer. Screening, treatment, and follow-up. NIH Consensus Development Panel on Ovarian Cancer. JAMA. 1995;273:491-7.

14. Goff BA, Mandel LS, Melancon CH, Muntz HG. Frequency of symptoms of ovarian cancer in women presenting to primary care clinics. JAMA. 2004;291:2705-12.

15. Padilla LA, Radosevich DM, Milad MP. Accuracy of the pelvic examination in detecting adnexal masses. Obstet Gynecol. 2000;96:593-8.

16. Bhosale $P$, Iyer $R$. Diagnostic imaging in gynecologic malignancy. Minerva Ginecol. 2008;60:143-54.

17. Heinz-Peer G, Memarsadeghi M, Niederle B. Imaging of adrenal masses. Curr Opin Urol. 2007; $17: 32-8$.

18. Dalrymple NC, Prasad SR, Freckleton MW, Chintapalli KN. Informatics in radiology (infoRAD): introduction to the language of three-dimensional imaging with multidetector CT. Radiographics. 2005;25:1409-28.

19. Buy JN, Ghossain MA, Sciot C, Bazot M, Guinet C, Prevot S, et al. Epithelial tumors of the ovary: CT findings and correlation with US. Radiology. 1991;178:811-8.

20. Byrom J, Widjaja E, Redman CW, Jones PW, Tebby $\mathrm{S}$. Can pre-operative computed tomography predict resectability of ovarian carcinoma at primary laparotomy? BJOG. 2002;109:369-75.

21. Mubarak F, Alam MS, Akhtar W, Hafeez S, Nizamuddin N. Role of multidetector computed tomography (MDCT) in patients with ovarian masses. Int J Womens Health. 2011;3:123-6.

22. Petru E, Schmidt F, Mikosch P, Pickel H, Lahousen M, Tamussino K, et al. Abdominopelvic computed tomography in the preoperative evaluation of suspected ovarian masses. Int J Gynecol Cancer. 1992;2(5):252-5.

23. Tsili AC, Tsampoulas C, Charisiadi A, Kalef-Ezra J, Dousias V, Paraskevaidis E, et al. Adnexal masses: accuracy of detection and differentiation with multidetector computed tomography. Gynecol Oncol. 2008;110:22-31.

24. Verit FF, Pehlivan M. Transvagınal ultrasound and computed tomography combined with ca- 125 
determinations in preoperative evaluation of ovarian masses in premenopausal women. Harran Üniv. T1p Fakültesi Dergisi. 2007;4(2):50-4.

25. Tsili AC, Tsampoulas C, Argyropoulou M, Navrozoglou I, Alamanos Y, Paraskevaidis E, et al. Comparative evaluation of multidetector CT and MR imaging in the differentiation of adnexal masses. Euro Radiology. 2008;18(5):1049-57.

26. Tsili AC, Dalkalitsis N, Paraskevaidis E, Tsampoulas K. Multi-detector CT features of benign adnexal lesions. Acad Radiol. 2010;17:31-8.

27. Firoozabadi RD, Zarchi MK, Mansuarian HR, Moghadam BR, Teimoori S, Naseri A. Evaluation of diagnostic value of CT scan, physical examination and ultrasound based on pathological findings in patients with pelvic masses. Asian Pacific J Cancer Prev. 2011;12:1745-7.

28. Gatreh-Samani F, Tarzamni MK, OladSahebmadarek, Dastranj, Afrough. Accuracy of 64 - multidetector computed tomography in diagnosis of adnexal tumors. J Ovarian Research. 2011;4:15.

29. Khattak YJ, Hafeez S, Alam T, Beg M, Awais M, Masroor I. Ovarian masses: is multi-detector computed tomography a reliable imaging modality? Asian Pacific J Cancer Prev. 2013;14(4):2627-30.

30. Jung SI, Park HS, Kim YJ, Jeon HS. Multidetector computed tomography for the assessment of adnexal mass: is unenhanced CT scan necessary? Korean J Radiol. 2014;15(1):72-9.

Cite this article as: Khalda E, Rahman H. Role of multi-detector computed tomography in the detection and differentiation of adnexal mass lesions. Int $\mathbf{J}$ Reprod Contracept Obstet Gynecol 2019;8:2725-31. 